\title{
Fucosyltransferase 4 and 7 mediates adhesion of non-small cell lung cancer cells to brain-derived endothelial cells and results in modification of the blood-brain-barrier: in vitro investigation of CD15 and CD15s in lung-to-brain metastasis
}

\author{
Samah A. Jassam ${ }^{1} \cdot$ Zaynah Maherally $^{1} \cdot$ Keyoumars Ashkan $^{2} \cdot$ Geoffrey J. Pilkington $^{1} \cdot$ Helen L. Fillmore ${ }^{1}$
}

Received: 15 February 2019 / Accepted: 6 May 2019 / Published online: 18 May 2019

(C) The Author(s) 2019

\begin{abstract}
Purpose Metastatic non-small cell lung (NSCLC) cancer represents one of the most common types of brain metastasis. The mechanisms involved in how circulating cancer cells transmigrate into brain parenchyma are not fully understood. The aim of this work was to investigate the role of fucosylated carbohydrate epitopes CD15 and sialyated CD15s in cancer adhesion to brain-derived endothelial cells and determine their influence in blood-brain barrier (BBB) disruption

Methods Three distinct, independent methods were used to measure brain endothelial integrity and include voltohmmeter $\left(\right.$ EVOM $\left.^{\mathrm{TM}}\right)$, impedance spectroscopy (CellZscope $\left.{ }^{\circledR}\right)$ and electric cell-substrate impedance sensing system (ECIS $\left.{ }^{\mathrm{TM}}\right)$. Two fucosyltransferases (FUT4 and 7) responsible for CD15 and CD15s synthesis were modulated in four human cancer cell lines (three lung cancer and one glioma).

Results Overexpression of CD15 or CD15s epitopes led to increase in adhesion of cancer cells to cerebral endothelial cells compared with wild-type and cells with silenced CD15 or CD15s $(\mathrm{p}<0.01)$. This overexpression led to the disruption of cerebral endothelial cell monolayers ( $\mathrm{p}<0.01)$. Knockdown of FUT4 and FUT7 in metastatic cancer cells prevented disruption of an in vitro BBB model. Surprisingly, although the cells characterised as 'non-metastatic', they became 'metastatic' -like when cells were forced to over-express either FUT4 or FUT7.

Conclusions Results from these studies suggest that overexpression of CD15 and CD15s could potentiate the transmigration of circulating NSCLC cells into the brain. The clinical significance of these studies includes the possible use of these epitopes as biomarkers for metastasis.
\end{abstract}

Keywords CD15 $\cdot$ CD15s $\cdot$ NSCLC $\cdot$ Brain metastasis $\cdot$ FUT4 $\cdot$ FUT7

\section{Introduction}

Samah A. Jassam and Zaynah Maherally shared the first authorship.

Electronic supplementary material The online version of this article (https://doi.org/10.1007/s11060-019-03188-x) contains supplementary material, which is available to authorized users.

Helen L. Fillmore

helen.fillmore@port.ac.uk

1 Cellular and Molecular Neuro-Oncology Research Group, School of Pharmacy and Biomedical Sciences, University of Portsmouth, White Swan Road, Portsmouth P01 2DT, UK

2 Neuro-Surgery, King's College Hospital, Denmark Hill, London SE5 9RS, UK
The majority of intracranial tumours are secondary metastases originating from primary non-central nervous system (non-CNS) cancers. 20-40\% of patients with systemic cancers develop secondary brain tumours $[1,2]$. The highest incidence of brain metastasis is seen in lung cancer patients (40-50\%) followed by breast (20-30\%) and melanoma $(5-10 \%)[1,3,4]$. As overall survival in patients with primary non-CNS cancers has improved, the incidence of metastasis to the brain has also increased, possibly due to circulating cancer cells which persist despite patient remission. The central nervous system (CNS) is considered a site of refuge as cancers are protected from most systemic therapies by the blood-brain barrier (BBB). The BBB is a 
dynamic and selective defensive barrier, which maintains a highly specific environment within the CNS by inhibiting both fluctuations of plasma components and entry of substances that may potentially cause brain toxicity [5]. Brain vascular endothelial cells are specific to the BBB with low pinocytosis and no fenestrations [6] and these cells may play a crucial role in the homing process of brain metastasis from breast cancer [7] and melanoma [8]. Although, leukocytelike mechanisms have been suggested in the extravasation process of some cancer cells $[9,10]$, the transmigration of cancer cells particularly to the brain has not been thoroughly investigated. Recently, we have characterised CD15 and CD15s (sialyl CD15) expression in non-small cell lung cancer cells and their potential importance in adhering to brain derived endothelial cells using a model that mimics physiological blood flow $[11,12]$. CD15 and CD15s, also known as Lewis ${ }^{\mathrm{x}}$ and sialyl Lewis ${ }^{\mathrm{x}}$ respectively, are classified as Type II Lewis antigens and are synthesized by specific fucosyltransferases [13]. To gain a better understanding on the role of CD15 and CD15s in lung cancer metastasis to the brain, two fucosyltransferases, FUT4 and 7, responsible for their corresponding synthesis were genetically modulated in non-small cell lung cancer cells. The effect of these genetically modified cells on endothelial cell adhesion and on integrity of an in vitro BBB model was then evaluated.

\section{Materials and methods}

\section{Cell culture}

The human cerebral microvascular endothelial cell line (hCMEC/D3) was donated by Professor Couraud (Institute of Cochin, INSERM, Paris, France) [14] and cultured in endothelial basal medium-2 (EGM-2) (Lonza, Germany) supplemented with $2 \%$ human serum (Sigma, UK). Primary NSCLC cells (COR-L105) were purchased from Sigma, UK; metastatic NSCLC cells from cervical lymph node (NCIH1299) from ATCC, UK and low-passage biopsy-derived brain-metastatic NSCLC cells, obtained from a patient with lung-brain metastatic cancer (SEBTA-001) as well as a biopsy-derived primary glioblastoma (GBM) cell line (UP007) both established "in house". Cell lines were maintained at $5 \% \mathrm{CO}_{2}$ and in a humidified atmosphere at $37{ }^{\circ} \mathrm{C}$. All lines were subjected to routine mycoplasma testing, and cell authentication [15].

\section{Antibodies}

Mouse monoclonal anti-CD15 (MEM-158) (Santa Cruz Biotechnology, USA) was used at the following dilutions: 1:100 for immunocytochemistry (ICC) and 1:10 for flow cytometry (FC). Mouse monoclonal anti-sialyl CD15 (BD Biosciences,
UK) was used at the following dilutions: 1:50 for ICC and 1:25 for FC. Secondary antibodies, fluorochrome-conjugated Alexa Fluor-488 and 568 IgM (Thermo Fisher Scientific, UK) were used for ICC and FC at 1:500.

\section{Overexpression and knockdown of CD15/FUT4 and CD15s/FUT7}

Overexpression of CD15 and CD15s was carried out by transfecting cell lines: SEBTA-001, NCI-H1299, CORL105 and UP-007 with human non-viral cDNA containing a unique construct of alpha $(1,3)$ fucosyltransferase (FUT4): (OriGene, USA, FUT4 (NM_002033) or alpha $(1,3)$ fucosyltransferase (FUT7) (OriGene, USA, FUT7 (NM_004479). cDNA constructs contained a GFP expression cassette. Transfection was carried out by using TurboFectin8.0 as per the manufacturer's protocol (OriGene, USA). In parallel, endogenous expression of CD15/FUT4 and CD15s/FUT7 were knocked down using four different human-FUT4 and FUT7 unique 29 mer shRNA constructs in pGF-V-RS GFP vectors (OriGene, USA).

\section{Immunocytochemistry}

Cells were seeded onto sterile coverslips at $1 \times 10^{3} /$ well overnight, fixed with $4 \%$ paraformaldehyde (PFA) (Sigma, UK) followed by three washes with phosphate-buffered saline (PBS) (Sigma, UK). Non-specific antigens were blocked with $10 \%$ goat serum (Sigma, UK) then incubated with the primary antibody for $1 \mathrm{~h}$ followed by $30 \mathrm{~min}$ incubation with their respective secondary antibody (Thermo Fisher Scientific UK). Hoechst Blue (Cell Signalling Technology, UK) was used as nuclear counterstain. Coverslips were examined using a Zeiss Axio fluorescence microscope and images were captured using a Volocity Image Analysis Software (V 5.2, Perkin Elmer).

\section{Confocal microscopy}

Images were obtained from a Zeiss LSM 510 Meta Axioskop 2 confocal microscope ( $\times 40$ and $\times 100$ objectives) using lasers with excitation wavelengths of $405 \mathrm{~nm}$ (blue), $488 \mathrm{~nm}$ (green) and $568 \mathrm{~nm}$ (red) and with diode, argon and $\mathrm{HeNe} 1$ lasers respectively. Identical settings were used to image negative controls in which primary antibody was replaced with non-specific Isotype.

\section{Flow cytometry analysis}

Cells were collected via gentle scraping, blocked in $2 \%$ goat serum/PBS (Sigma, UK) and primary antibodies applied while non-specific IgM isotype was added to the negative control and incubated for $30 \mathrm{~min}$. Cells were then washed 
and secondary antibodies (ThermoFisher Scientific, UK) applied for $15 \mathrm{~min}$ followed by more washes before transferring to fluorescence-activated cell sorting (FACS) tubes (BD Biosciences, UK) containing $5 \mu \mathrm{L}$ of Propidium iodide (PI) (Cell Signalling Technology, UK). Samples were analysed using a 4-color-multiparameter FACS Calibur (BD Biosciences-UK). Each experiment was repeated three independent times in triplicate. Data were represented as percentage of positive cell population.

\section{Adhesion assay}

An adhesion assay kit, CytoSelect Tumor-Endothelium (Cell Biolabs, UK) was used [11, 12]. Briefly, $1 \times 10^{6}$ brain endothelial cells/well were seeded onto a sterile surface coated with fibronectin $(10 \mathrm{mg} / \mathrm{mL})$. Cells were grown to form a complete monolayer. Cancer cells, labelled with a green fluorescent dye (Cell Biolabs, UK), were seeded on surface of the activated (with $25 \mathrm{pg} / \mathrm{mL}$ TNF- $\alpha$ ) hCMEC/ D3 monolayer and incubated for $90 \mathrm{~min}$. Non-adherent cells were washed thoroughly with pre-warmed PBS. Representative adherent cells were assessed using a POLARstar OPTIMA microplate reader (BMG LABTECH, UK). The experiment was repeated 3 independent times in triplicate.

\section{Trans-endothelial migration studies}

\section{Voltohmmeter (EVOM ${ }^{\mathrm{TM}}$ )}

Polycarbonate membrane Transwell inserts (24 well, $8.0 \mu \mathrm{m}$ pore size) (Thermo Fisher, UK, UK) were pre-coated with $10 \mu \mathrm{g} / \mathrm{mL}$ human fibronectin (Sigma, UK) prior to addition of medium supplemented with TNF- $\alpha(25 \mathrm{pg} / \mathrm{mL})$ and $1 \times 10^{5}$ cells/well of hCMEC/D3 to apical side of the inserts. Readings were recorded using a voltohmmeter $\left(\mathrm{EVOM}^{\mathrm{TM}}\right)$ (World Precision Instruments, USA). When resistance reached a plateau, $2.0 \times 10^{4}$ cells/well were added on top of the hCMEC/D3 monolayer. Five readings were recorded per day and resistance measurement monitored for a further 5-day period and Ohm's law applied.

\section{Impedance spectroscopy (CellZscope ${ }^{\circledast}$ )}

hCMEC/D3 cells $\left(1 \times 10^{5} /\right.$ well $)$ were seeded on fibronectincoated $(10 \mu \mathrm{g} / \mathrm{mL})$ polycarbonate Transwell inserts ( 24 well, $8.0 \mu \mathrm{m}$ pore size) (Thermo Fisher, UK), placed in the CellZscope ${ }^{\circledR}$ module and incubated in a sterile, humidified, 37 ${ }^{\circ} \mathrm{C}$ and $5 \% \mathrm{CO}_{2}$ incubator. Resistance values were recorded using an automated cell monitoring system, CellZscope ${ }^{\circledR}$ (nanoAnalytics, UK) until they reached a plateau wherein $2.0 \times 10^{4}$ cells/well were added on top of the hCMEC/D3 monolayer. TER values, expressed in $\Omega \cdot \mathrm{cm}^{2}$, were recorded in real-time over a 5-day period post-addition of cancer cells. All experiments were carried out in triplicate and repeated at least three times.

\section{Electric cell-substrate impedance sensing system $\left(\mathrm{ECIS}^{\top \mathrm{T}}\right)$}

ECIS arrays (8W10E+, ibidi, Germany) were stabilised with L-cysteine (10 nM; 10 min incubation; Sigma, UK), washed in Hank's balanced salt solution (Fisher, UK) and coated with $10 \mathrm{mg} / \mathrm{mL}$ fibronectin for $2 \mathrm{~h}$ followed by seeding of $7.5 \times 10^{4}$ cells/chamber of hCMEC/D3 in media supplemented with TNF- $\alpha(25 \mathrm{pg} / \mathrm{mL})$. Resistance was monitored using an ECIS Z $\theta$ (Applied Biophysics, USA) system until a peak resistance was achieved. $2.0 \times 10^{4}$ of cancer cells were then added onto the endothelial cell monolayer. Resistance values were obtained in $\Omega$. The confluency of the endothelial cell monolayer was also evaluated by using an Olympus 1 X71 inverted phase contrast microscope.

\section{Statistical analysis}

All experiments were performed three times in triplicate, and data expressed as + SE. Statistical analyses were performed using One-way ANOVA followed by Tukey's multiple comparison post hoc tests using Graph Pad Prism 6 software for analysis.

\section{Results}

Three NSCLC cell lines and one GBM cell line (SEBTA001, NCI-H1299, COR-L105 and UP-007) were transfected with either a full-length FUT4-GFP for CD15-overexpression or FUT4 shRNA constructs for CD15-knockdown. CD15 protein expression was examined using ICC, and FC analyses (Fig. 1a, c). The highest levels of CD15 protein expression were seen on the surface of the NSCLC metastatic cell lines (NCI-H1299 and SEBTA-001), with lower cell surface expression in the non-metastatic cell lines (COR-L105 and UP-007), consistent with our previous report [11]. Expression of CD15 in cells transfected with FUT4 shRNA constructs demonstrated knockdown of CD15 expression (Fig. 1a, c). shRNA knockdown of FUT4 resulted in a significant decrease in CD15 positive cells in SEBTA001, NCI-H1299, COR-L105, and UP-007 cells (Fig. 1c; $\mathrm{p}<0.01)$. In cell lines transfected with FUT4 cDNA constructs, there was an increase in CD15 expression (Fig. 1a, c). In SEBTA-001 CD15 expression increased from 54.7 to 92\%, in NCI-H1299 from 76 to 90\%, in COR-L105 from 23 to $89 \%$ and in UP-007 from 13.4 to $84 \%$ (Fig. 1c; p < 0.01 ).

Using the same four cell lines and methods mentioned above, the effects of either over-expressing FUT7 or silencing FUT7 on CD15s expression was determined. The 
FUT4/CD15

A
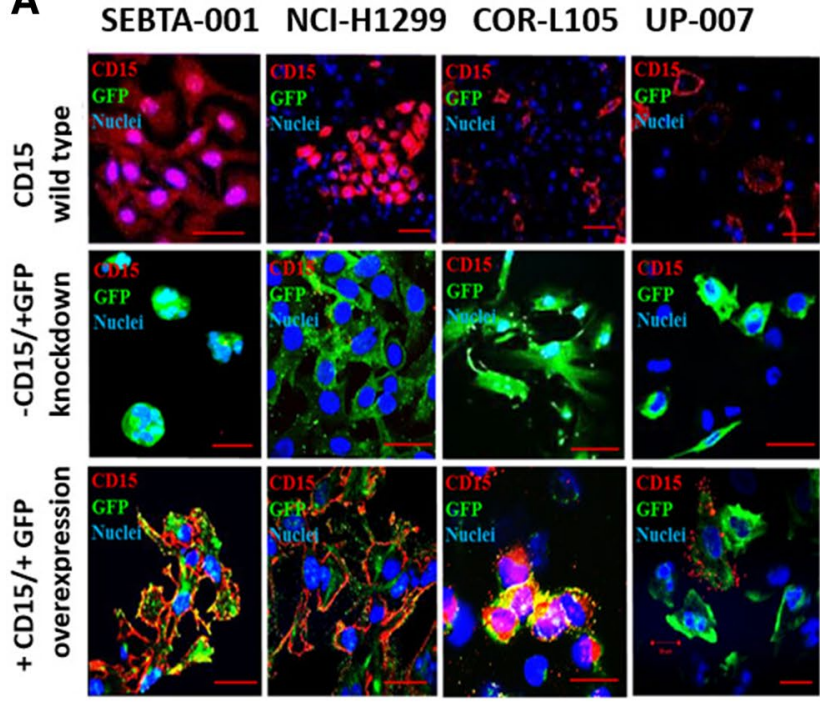

B

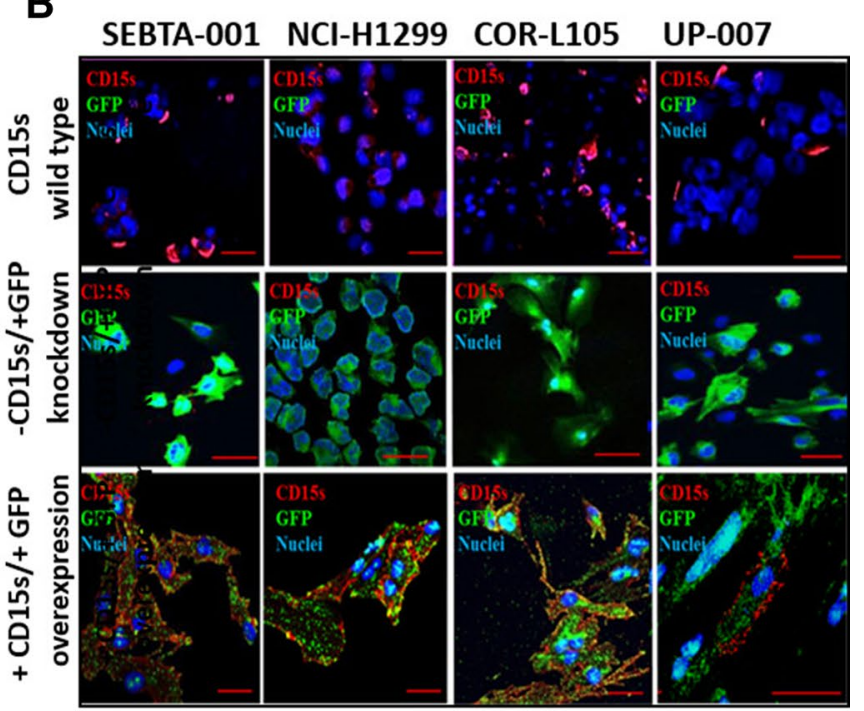

C

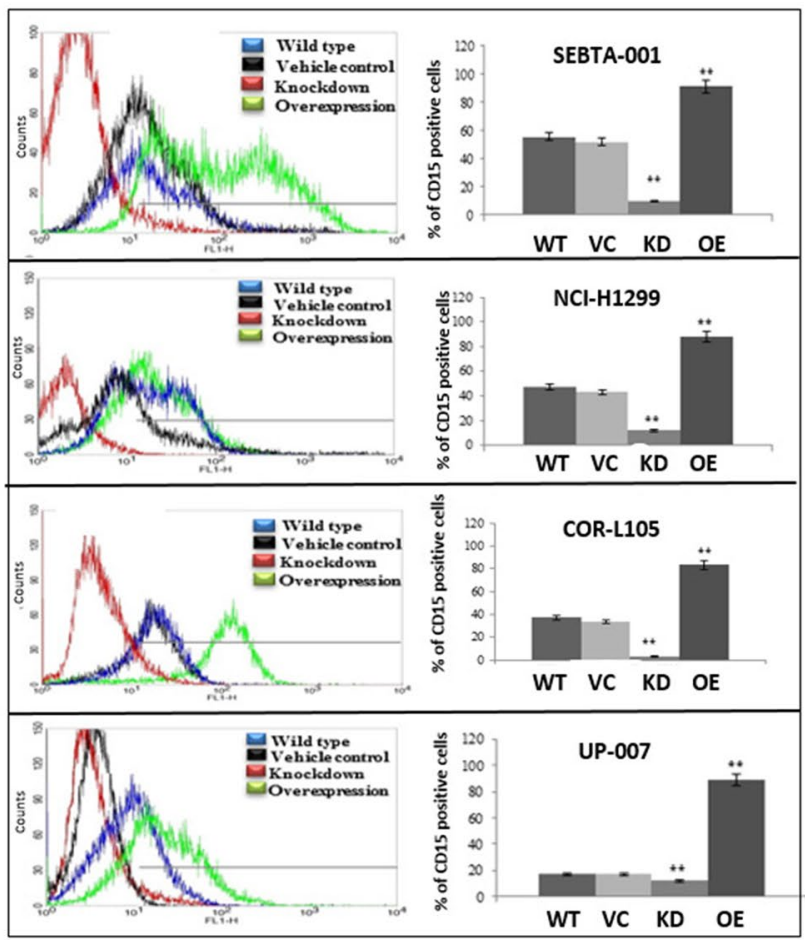

Fig. 1 CD15 and CD15s expression in FUT4/FUT7-(cDNA and shRNA) transfected cells. Target cells (SEBTA-001, NCI-H1299, COR-L105 and UP-007) were transfected, either with plasmids containing FUT4 or FUT7-cDNA to induce CD15 or CD15s overexpression, or with FUT4 or FUT7-shRNA constructs for CD15 or CD15s knockdown. a, c FUT4/CD15. b, d FUT7/CD15s. a, b Representative confocal images showing localisation of a CD15 or b CD15s (red) cells grown on coverslips. c, d Representative FC analysis of CD15
D

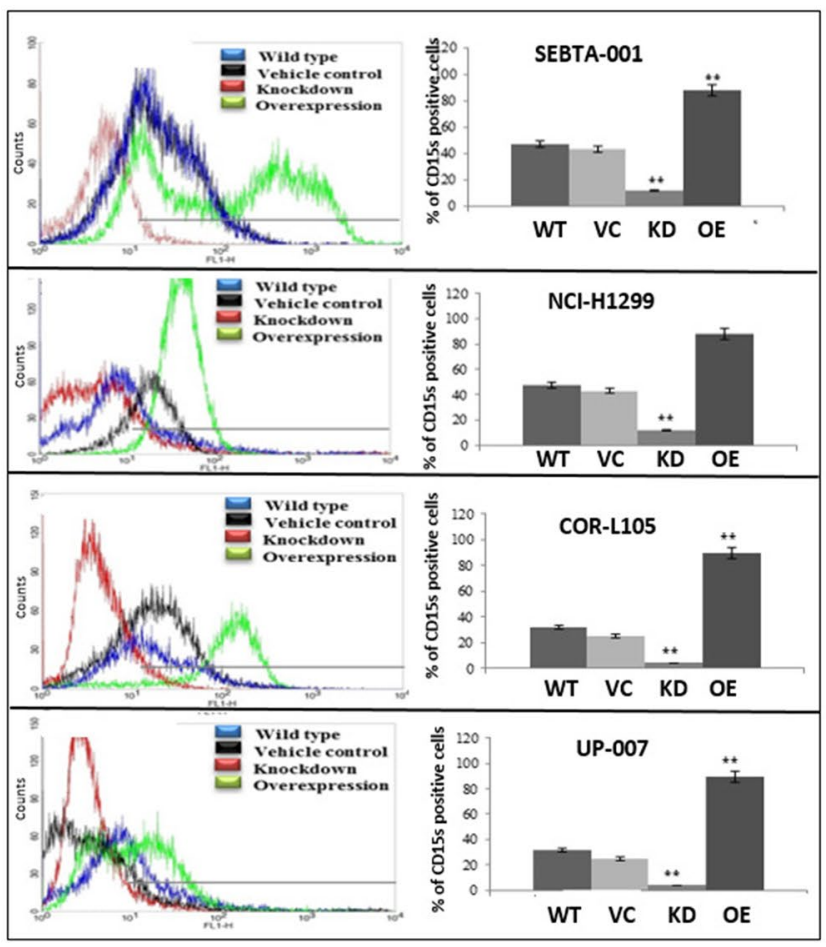

or CD15s expression. The histograms show percentages of CD15/ $\mathrm{CD} 15 \mathrm{~s}$ positive cells in wild type controls (blue) where no treatment applied, vehicle controls were transfected with non-coding plasmid (black), cells transfected with shRNA for FUT4 or FUT7 (red) and in cells transfection with cDNA for FUT4 or FUT7 (green). ( $* * \mathrm{P}<0.01$ ) difference compared to the wild type. $\mathrm{N}=3$. No treatment was applied to wild type controls and vehicle controls were transfected with non-coding plasmids. Scale bar $=20 \mu \mathrm{m}$ 
absence of CD15s was noted in cells treated with FUT7shRNA (Fig. 1b, d). Expression of CD15s was significantly decreased post-transfection with FUT7-shRNA in all the cell lines: in SEBTA-001, NCI-H1299, COR-L105, and UP-007 ( $p<0.01)$. FC analyses results showed a significant increase in CD15s expression on cells when transfected with FUT7-cDNA. The number of CD15s positive cells increased from 55 to $88 \%$ in SEBTA-001, 51-78\% in NCI-H1299, $33-53.8 \%$ in COR-L105 and 17-45\% in UP-007 (Fig. 1d, $\mathrm{p}<0.01)$. These results suggest a strong correlation exists between genetic manipulation of FUT4/FUT7 and CD15/ CD15s expression.

Following genetic manipulation of FUT4/CD15 or FUT7/ CD15s, changes in cell adhesion to an endothelial cell monolayer hCMEC/D3 was investigated. SEBTA-001 and NCIH1299 showed the highest number of adherent cells compared to COR-L105 and UP-007 (Fig. 2a-h). Adhesion of cancer cells was significantly reduced following the knockdown of FUT4/CD15 and the number of adherent cells were less compared to the wild type $(\mathrm{P}<0.01)$ (Fig. 2a-d). A significant reduction was observed after knockdown of FUT7/ CD15s in SEBTA-001 (2.5-fold decrease) and a 2.7-fold decrease in NCI-H1299, a 2.8-fold decrease in COR-L105 and a 2.8-fold decrease in UP-007 cells compared to the wild type $(\mathrm{P}<0.01)$ (Fig. 2e-h). Following the overexpression of FUT4/CD15 in SEBTA-001, NCI-H1299, COR-L105 and UP-007, a significant increase in adhesion (twofold) was seen compared to the adhesion of wild type control of the same cell line (Fig. 2a-d, p <0.01). Similar results were observed for FUT7/CD15s (Fig. 2e-h, p <0.01).

We next measured changes in transendothelial electrical resistance (TEER) using three independent technologies [16]. The EVOM ${ }^{\mathrm{TM}}$ technology, often referred to as the 'chop-stick' method was used to collect data every $2 \mathrm{~h}$ for $48 \mathrm{~h}$. When wildtype SEBTA-001 and NCI-H1299 cells were added to an intact hCMEC/D3 monolayer, a decrease in resistance was observed over time and at the $48 \mathrm{~h}$ time point, this was significant (Fig. 3a, b, e, f; p $<0.01$ ), whereas wildtype COR-L105 and UP-007 cells did not cause a change in resistance over time (Fig. 3c, d, g, h). Knock-down experiments targeting either FUT4 or FUT7 in SEBTA-001 and NCI-H1299 cells attenuated the changes in resistance seen with respective wildtype cells (Fig. 3a, b, e, f).

Enhanced expression of FUT4 or FUT7 in all cell lines resulted in a significant decrease in TEER values at $48 \mathrm{~h}$ (Fig. 3a-h; p < 0.05). Although there appears to be a trend towards a decrease in TEER values in UP-007 cells transfected with FUT4, UP-007 cells transfected with FUT7, did result in a significant decrease in TEER values at the $48 \mathrm{~h}$ time point ( $<<0.05$; Fig. 3d vs. h).

The CellZscope ${ }^{\circledR}$ methodology offers a real-time acquisition of resistance data. Addition of wildtype SEBTA-001 and NCI-H1299 to an intact monolayer of hCMEC/D3 cells resulted in a significant decrease in Transendothelial Resistance TER values over time compared to the non-metastatic cell lines, COR-L105 and UP-007 (Fig. 4a-h; p $<0.05$. Bar graphs represent $24 \mathrm{~h}$ ). The silencing of either FUT4 or FUT7 resulted in the decrease in the ability of SEBTA-001 and NCI-H1299 to affect hCMEC/D3 monolayer resistance (Fig. 4a-h). The overexpression of either FUT4 or FUT7 in cell lines tested (except for UP-007 transfected with FUT4), when plated onto a stable hCMEC/D3 monolayer, resulted in a significant reduction in TER values over time (Fig. $4 \mathrm{a}-\mathrm{h}$; $\mathrm{p}<0.05$ ).

ECIS $^{\mathrm{TM}}$ results were consistent with EVOM $^{\mathrm{TM}}$ and CellZscope ${ }^{\circledR}$ findings, and a strong association was noted between the expression of FUT4/CD15 and FUT7/CD15s and the decrease in the resistance of the brain endothelial monolayer. ECIS graphs and microscopic images demonstrate the changes in resistance of the endothelial cell monolayer, preand 24 h-post-addition of cancer cells. Addition of wildtype SEBTA-001 and NCI-H1299 cells led to a significant reduction in resistance from 232 to $168 \Omega$ and from 224 to $190 \Omega$ $(\mathrm{p}<0.05)$ respectively (Fig. 5a-d). In support of endothelial layer disruption, microscopic images show wide gaps in the endothelial monolayer barrier (arrows; Fig. 5). Knockdown of CD15 and CD15s in SEBTA-001 and NCI-H1299 did not result in significant changes to resistance. In addition, images of monolayer show a well-structured endothelial monolayer (Fig. 5d). Similar to the other methods used for measuring BBB integrity, both CD15 and CD15s overexpressing cells led to dramatically reduction in resistance (Fig. 5a-d; Supplemental Fig. 1a-d).

\section{Discussion}

CD15 and CD15s, well characterized for their involvement in the homing process of leukocytes, are correlated with cancer progression and metastasis in non-CNS cancers [17-19]. In previous studies, we showed that CD15 and CD15s are involved in the adhesion of cancer cells to brain endothelium via CD62E interaction [11, 12]. Here, we wanted to further investigate the role of CD15 and CD15s on the potential transmigration of cancer cells across an intact brain endothelial monolayer by modulating CD15 and CD15s expression by targeting the FUT4 and FUT7 genes.

CD15 and CD15s biosynthesis is regulated by fucosyltransferase enzymes (alpha-1,3 FUTs); FUT3,-4,-5,-6,-7 and -9 , which are spatial and temporally regulated and which expression appears to be cell/tissue specific [20-23]. Overexpression of FUT4 leads to the increase in CD15 expression and correlates with metastasis in colorectal cancer [24]. Conversely, FUT4 knockdown is reported to reduce the expression of CD15 in promyelocytes and monocytes [25]. 


\section{FUT4/CD15}

A
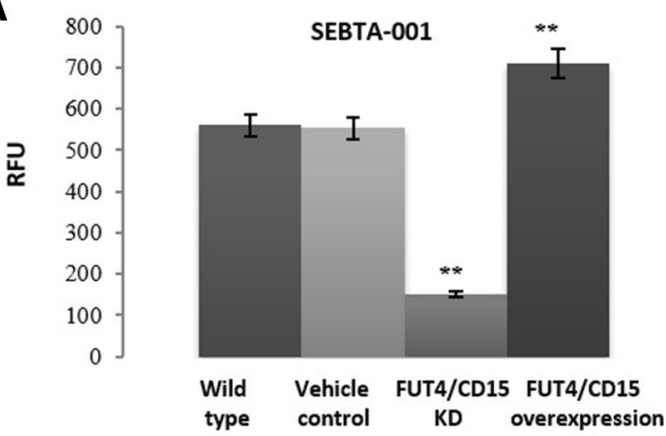

C

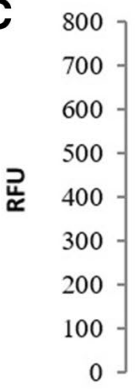

COR-L105

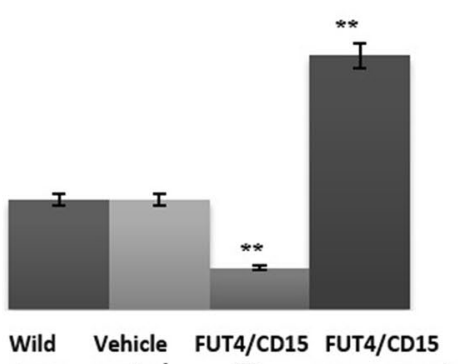

B

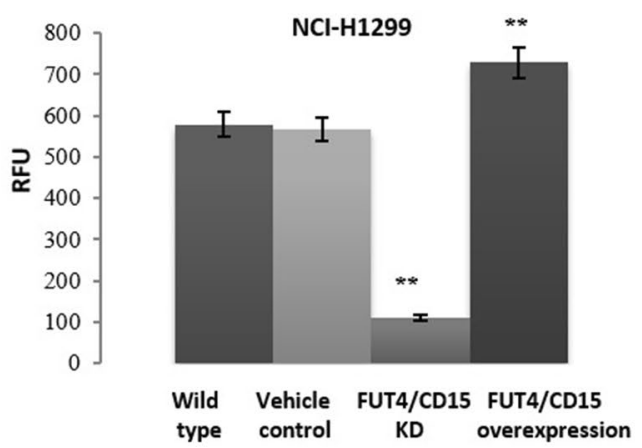

D

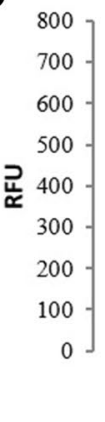

UP-007

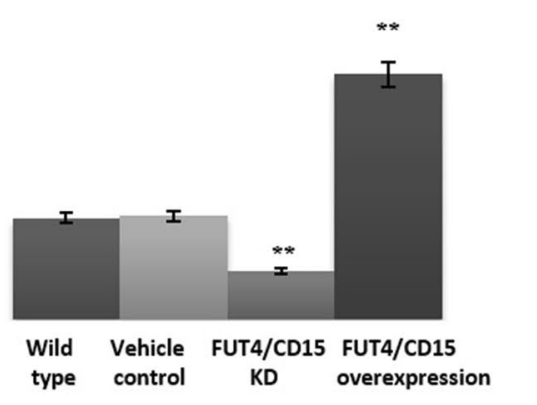

FUT7/CD15s

$\mathbf{E}$

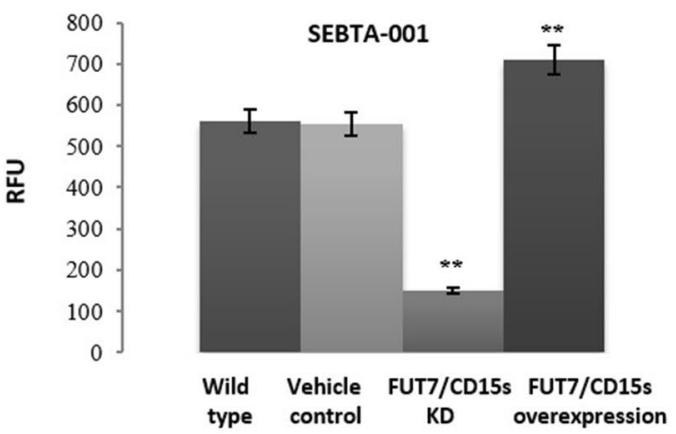

G

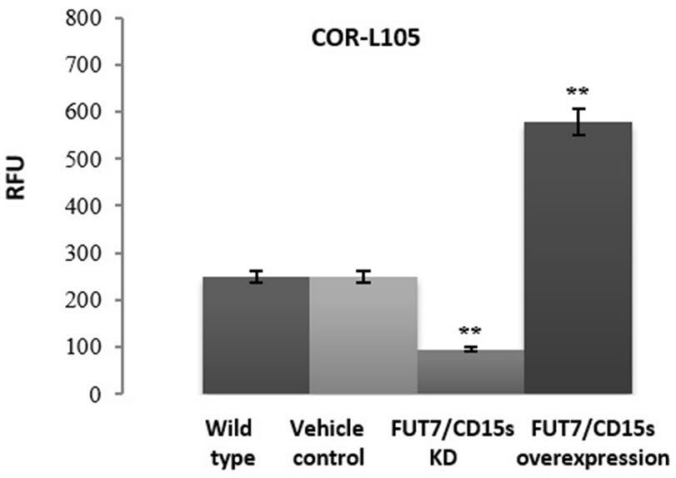

Fig. 2 Adhesion of cancer cells following genetic manipulation of CD15 and CD15s. Adhesion of cancer cells following FUT4/CD15 and FUT7/CD15s manipulation on metastatic NSCLC SEBTA-001 $(\mathbf{a}, \mathbf{e}), \mathrm{NCI}-\mathrm{H} 1299(\mathbf{b}, \mathbf{f})$ and non-metastatic NSCLC: COR-L105 (c,

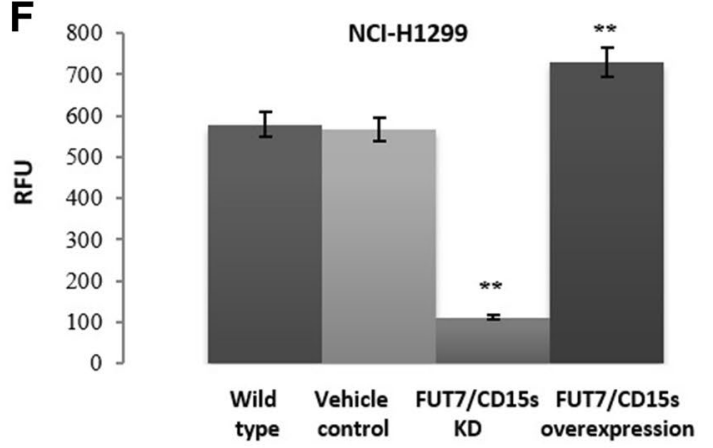

H

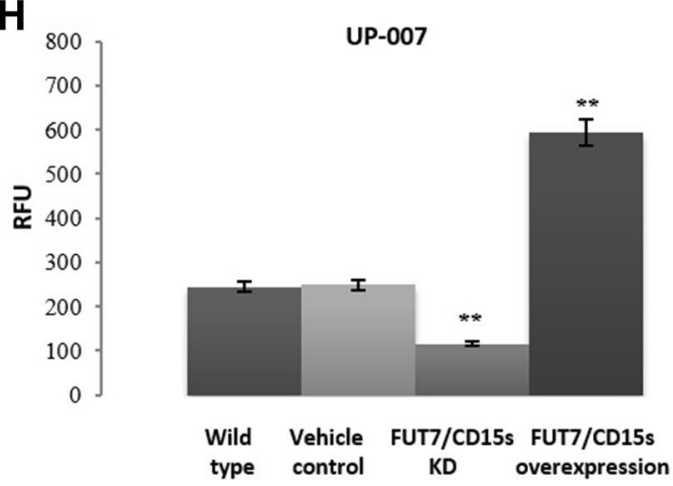

g), UP-007 (d, h). Cancer cells were incubated for 90 min on a monolayer of activated brain endothelial cells (hCMEC/D3). Non-adherent cancer cells were washed off followed by lysis of cells and quantified via a microplate reader at $480-520 \mathrm{~nm}$. $(* * \mathrm{P}<0.01), \mathrm{N}=3$ 


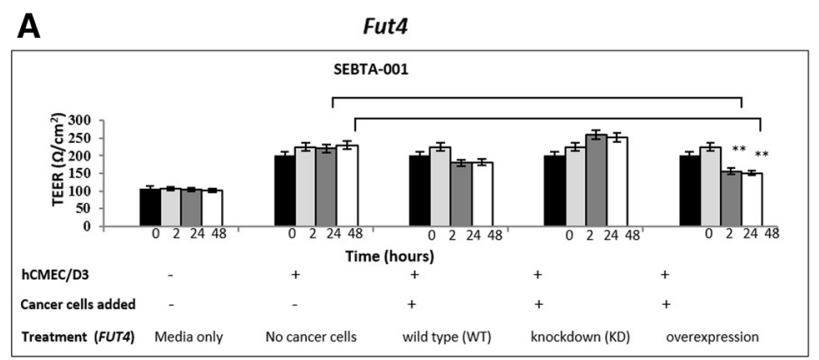

B

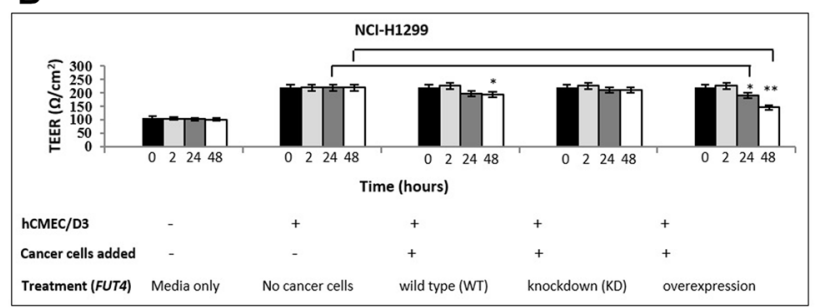

C

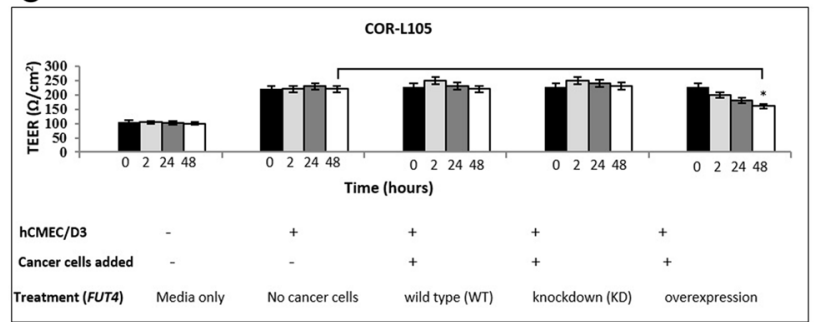

D

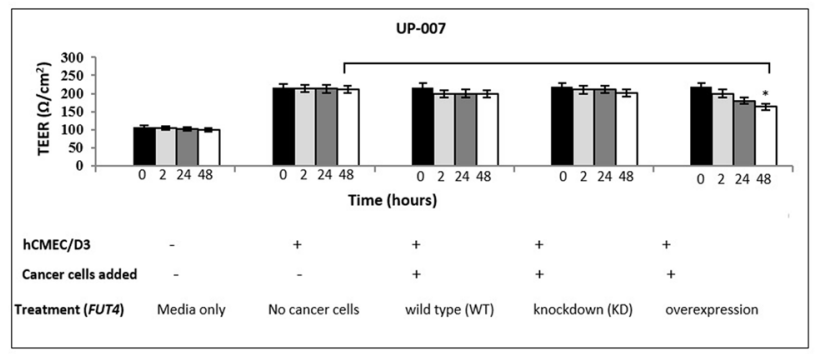

Fig. 3 Effects of FUT4/CD15 and FUT7/CD15s modulation on intact brain endothelial monolayer assessed by EVOM ${ }^{\mathrm{TM}}$. Resistance of hCMEC/D3 monolayer in response to cancer cells following FUT4/ CD15 (a-d) and FUT7/CD15s (e, f) genetic manipulation on SEBTA001 (a, e), NCI-H1299 (b, f), COR-L105 (c, g) and UP-007 (d, h). TEER values decreased when cell lines (except for UP-007) were transfected with FUT4/CD15 were placed on an established endothe-

FUT4 and FUT7 knockdown was shown to significantly reduce the trafficking of leukocytes and lymphocytes [26]. FUT overexpression in general has been correlated with poor prognosis and metastasis in prostate cancer [27-29] as well as lung cancer [30].

Overexpression of FUT4/CD15 led to an increase in endothelial cell adhesion in all studied cancer cells and FUT4/CD15 knockdown resulted in decreased adhesion. These findings are in agreement with that of Yang et al. [31] where overexpression of FUT4 correlated with high metastatic potential in breast cancer cells and implicated

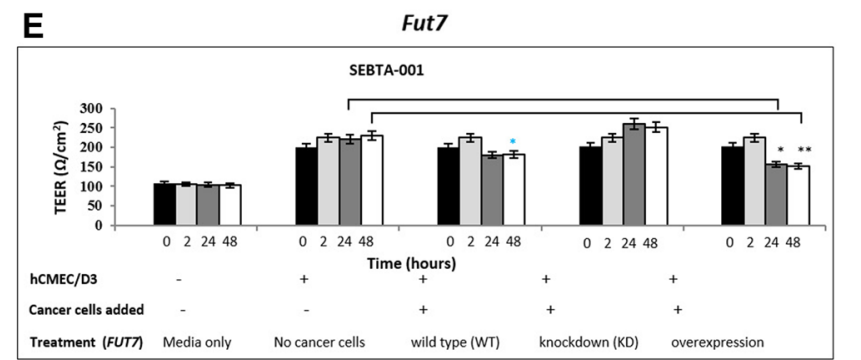

$\mathbf{F}$

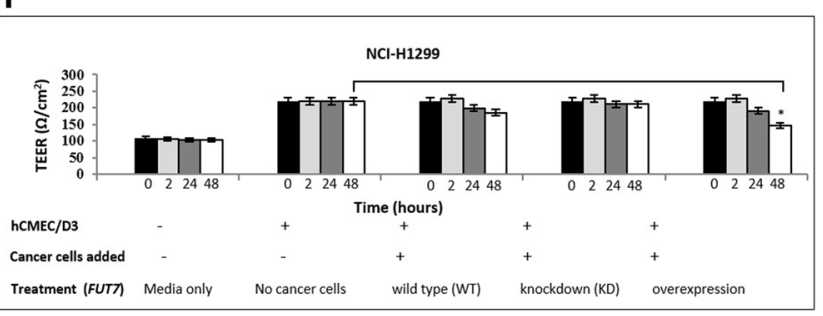

G

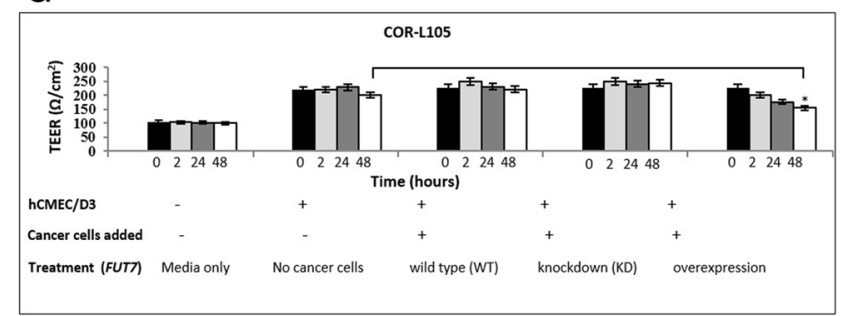

H

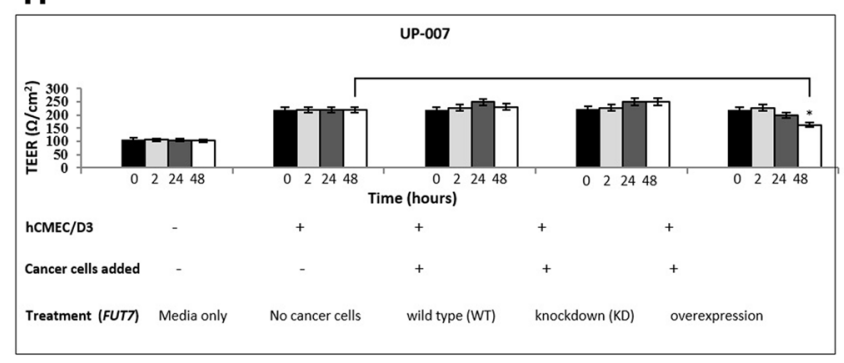

lial cell layer. Similar data were observed for cells transfected with FUT7/CD15s. The black bars represents the resistance of endothelial monolayer before adding cancer cells, light-grey bars to TEER values $2 \mathrm{~h}$ post-addition of cancer cells, dark-grey bars to TEER values 24-h post-addition of cancer cells and white bars to TEER values 48 -h post addition of applying cancer cells. a-c; $* \mathrm{p}<0.01, * * \mathrm{p}<0.05 . \mathrm{N}=3$

in adhesion and invasion. Knockdown of FUT4/CD15 and FUT7/CD15s significantly reduced the number of adherent metastatic and primary lung cancer cells as well as GBM cells to brain endothelium.

The findings related to the GBM cell line are very interesting. GBM rarely metastasises to other organs [32]. Martin et al. in 1995 [32] suggested that the absence in CD15 expression in GBM cells may contribute to the failure of GBM cells to metastasise due to the inability for glioma cells to attach to brain endothelial cells. The expression dynamics of CD15/CD15s in GBM could help to explain 
A

Fut4
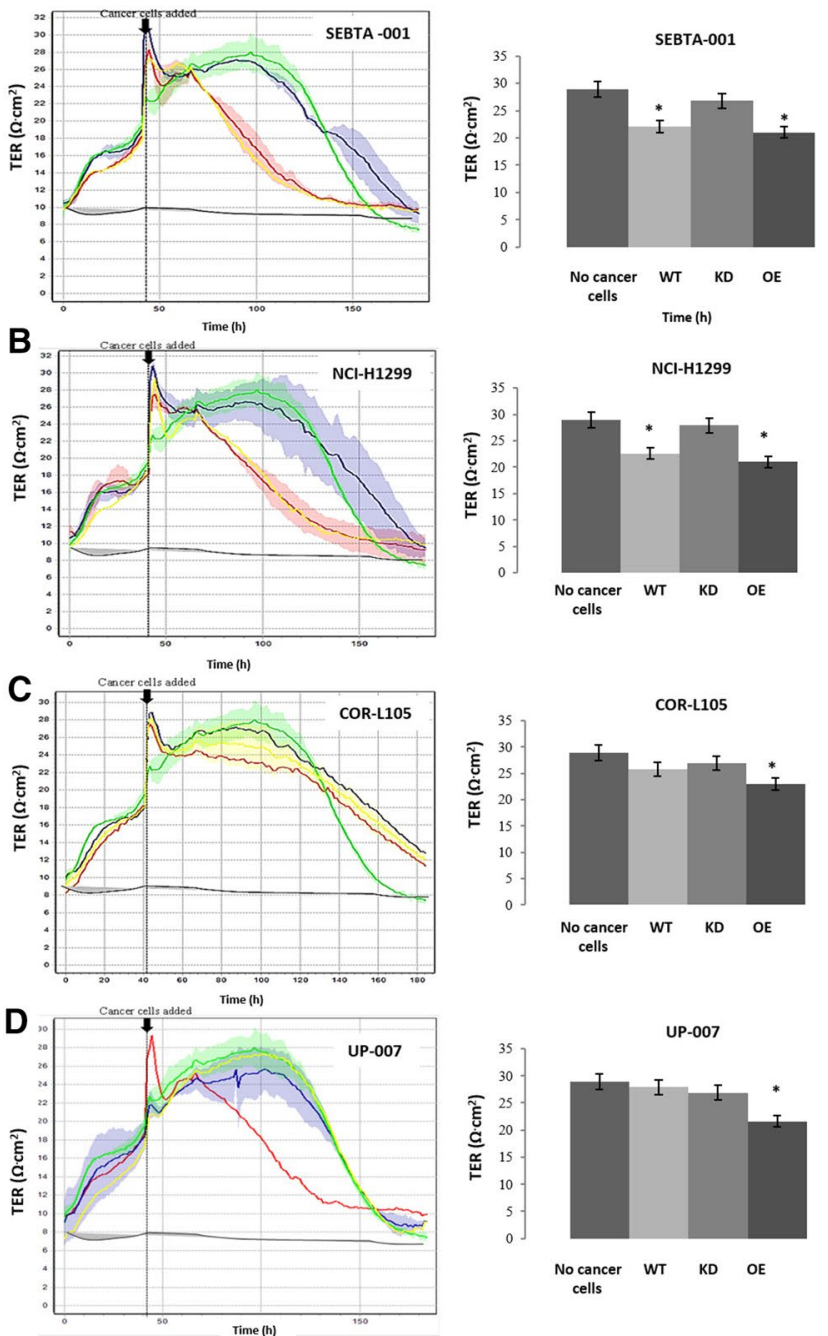

Fig. 4 Effects of FUT4/CD15 and FUT7/CD15s modulation in cells on brain endothelial monolayer measured in real-time. Representative histograms depicting the changes in TEER values, pre and post addition of cancer cells using the automated system (CellZscope $\left.{ }^{\circledR}\right)$. The green line represents endothelial cells (control) with no addition of cancer cells, red line represents FUT4/CD15 overexpressor

the rarity of GBM extraneural metastasis. Could CD15/ CD15s expression dynamics be a mechanism in which secondary lesions appear remote from the initial presentation site? GBM cells which have exited and entered the peripheral circulation may at least in theory, re-enter the brain to set up a second lesion site. This re-entry phenomenon if proven true may offer a second possibility underlying the observation of multiple GBM lesions currently believed to be due to invasion of GBM rogue cells. At this stage, this theory needs to be further investigated.
E

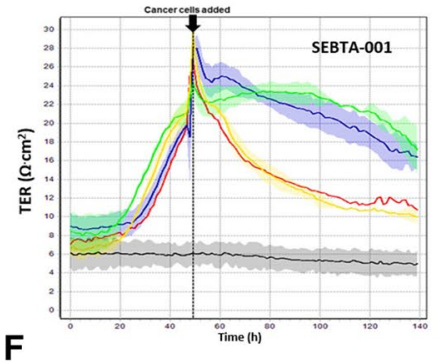

Fut7
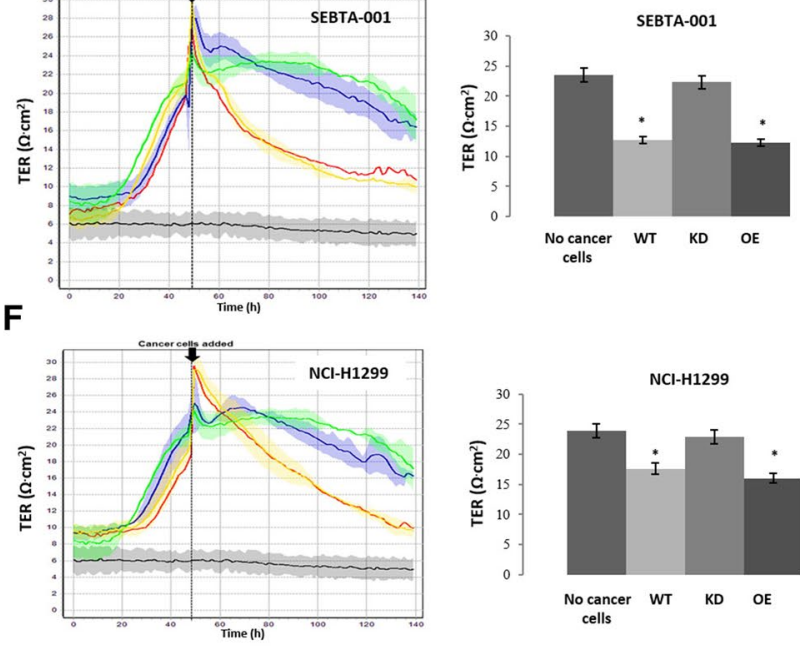

G
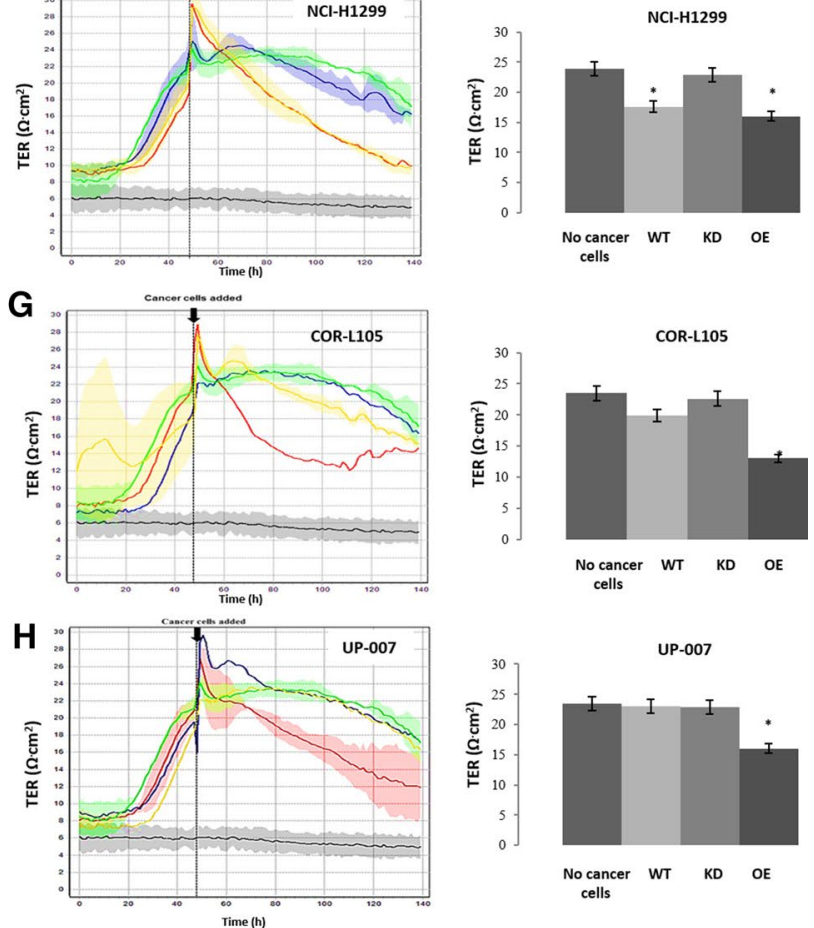

cells (a-d) or FUT7/CD15s (e-h), blue line represents FUT4/CD15 shRNA transfected cells (a-d) or FUT7/CD15s shRNA cells (e-h), yellow line representing the wild type and black line representing the blank. Bar chart represents quantified TER values $(* \mathrm{P}<0.05), \mathrm{N}=3$. $W T$ wild type, $K O$ knockdown, $O E$ overexpression

While our data suggests a role of CD15/s in NSCLC metastasis to brain, there remain questions into mechanisms for trans-endothelial transmigration of cancer cells. A likely scenario involves a bi-directional initiation of signalling cascades leading to the orchestration of processes such as the release of enzymes responsible for the breakdown of basal lamina for example. All of which underpin migration and invasion into the brain. This is followed by colonisation of the brain by interplay between tumour cells and the brain micro-environment. 
A

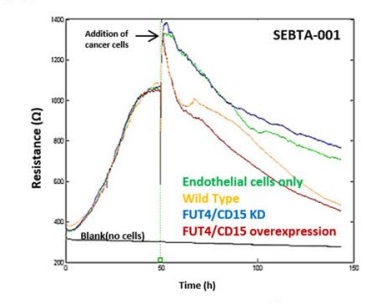

FUT4/CD15

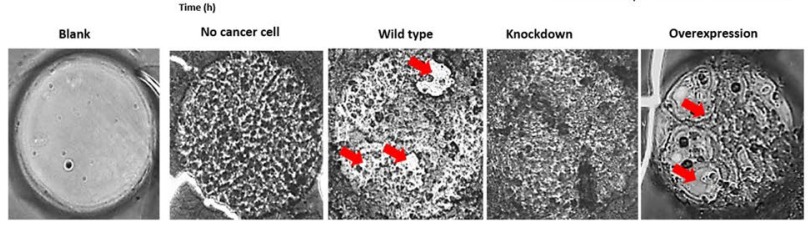

B
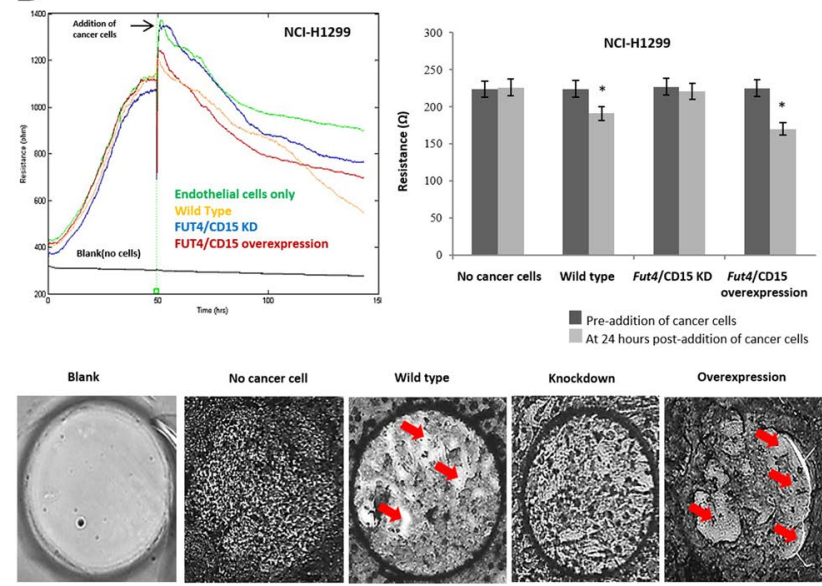

No cancer cell
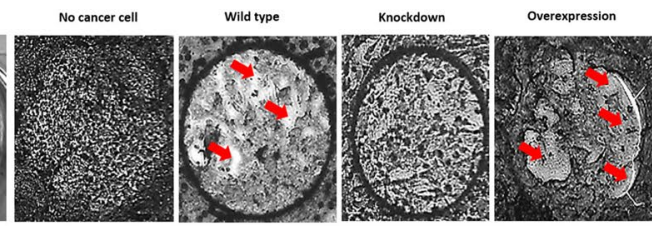

Fig. 5 FUT4 and FUT7 knockdown in metastatic cancer cells prevents disruption of intact brain endothelial monolayer. FUT4 in SEBTA-001 (a) and NCI-H1299 (b). FUT7 in SEBTA-001 (c) and NCI-H1299 (d). a-d Representative histograms showing resistance of endothelial barrier in real time, pre and post addition of cancer cells measured by (ECIS ${ }^{\mathrm{TM}}$ ). Green line represents control (no cancer cells), Red line represents FUT4/CD15 (a, b) or FUT7/CD15s (c, d) overexpressing cancer cells, blue line represents knockdown cell lines FUT4/CD15 (a, b) or FUT7/CD15s (c, d), yellow line represents

Recently Zhao et al. [33], identified a prognostic signature from a comprehensive bioinformatic analyses of 500 lung adenocarcinoma samples. One of the 20 genes highlighted, FUT4, was found to be one of the most significant genes associated with poor survival. It would be interesting to determine if the FUT genes are associated with brain metastases. Another clinical aspect of our studies is the potential to use these epitopes as therapeutic targets to prevent cerebral metastases of NSCLC by blocking adhesion to brain endothelial cells. While there are several temporal doorways for therapeutic targeting of metastatic spread, from intravasation to development of secondary lesions in the brain, it is generally agreed that once in the brain, the lesion is protected from the full benefits of treatment due to the blood brain barrier. With the advances in biomarkers and in liquid biopsies of
C

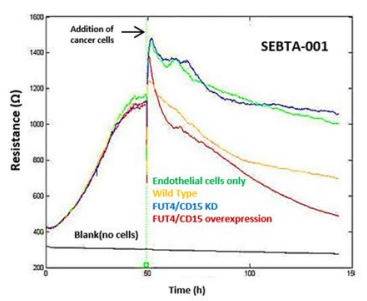

FUT7/CD15s

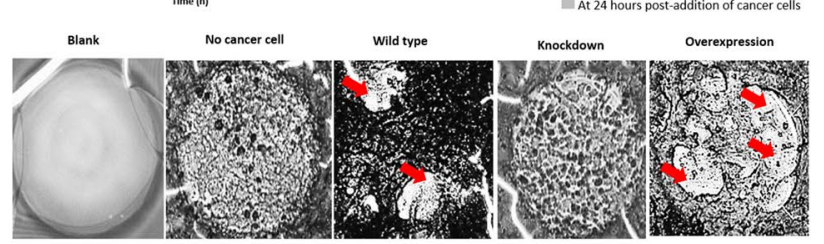

D
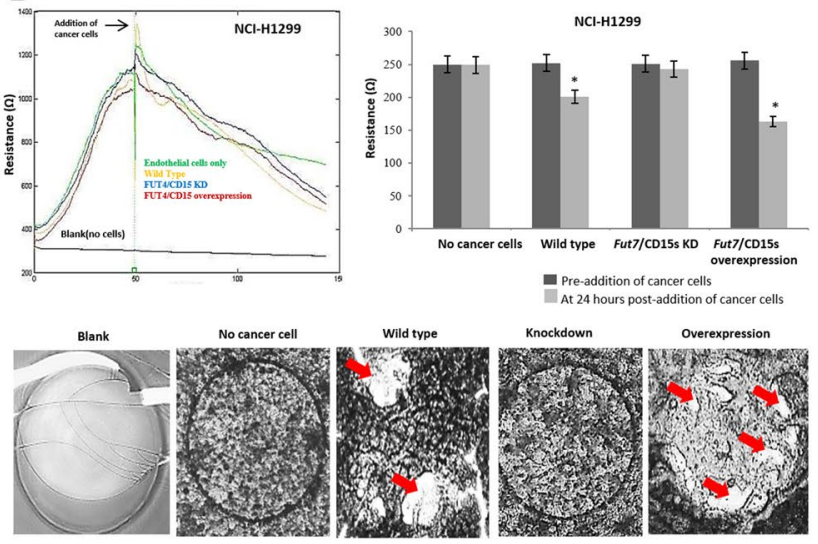

wildtype cancer cells and black line represents blank no cells. (a-d) Bar charts represent quantified resistance values at 24-h pre-postaddition of cancer cells $(* \mathrm{P}<0.05)$. Lower panel a-d) Representative microscopic images demonstrating the changes of integrity in brain endothelial monolayer, at time point of $24 \mathrm{~h}$ post-addition of cancer cells. Red arrows point to areas of endothelial cell monolayer disruption. Images were obtained using a phase contrast microscope $(\times 4)$. All studies $\mathrm{N}=3$

circulating cancer cells, the possible use of these epitopes as biomarkers for metastasis merits further study.

\section{Conclusions}

This study sheds light on two fucosyltransferases (FUT4 and 7) and the role of CD15 and CD15s expression in adhesion to and disruption of cerebral endothelial cell monolayers. Our data demonstrate that blocking of CD15 or CD15s expression weakens lung cancer cell adhesion and impedes the disruption of an in vitro model of a BBB model. Surprisingly, over-expression of these epitopes by inducing FUT4 or FUT7 in cell lines not known to be 
'metastatic' become 'metastatic-like' in regards to disruption of the BBB. While exciting, future in vivo studies are needed to confirm our in vitro observations.

Acknowledgements We acknowledge members of the Brain Tumour research Centre, at the University of Portsmouth for their support in this work. Special thanks to Katie Loveson and James Smith for helpful discussions with the writing of the manuscript and to Samantha Higgins for her editorial assistance.

Author contributions SJ, ZM and HLF designed the research and analysed data. SJ performed experiments. KA provided tissue and reviewed work. SJ, ZM, GJP and HLF wrote the paper. All authors have read and approved the manuscript.

Funding SAJ was funded by the High Committee of Education Development in Iraq (HCED-IRAQ). ZM was funded by a grant from Animal Free Research UK to GJP. HLF was supported by Brain Tumour Research.

\section{Compliance with ethical standards}

Conflict of interest The authors declare that they have no conflict of interest.

Ethical approval All cell lines established in house were established in accordance with the National Research Ethics Service (NRES) instructions and conformed to the Ethics permission LREC00-17,311/ SC/0048.

Open Access This article is distributed under the terms of the Creative Commons Attribution 4.0 International License (http://creativeco mmons.org/licenses/by/4.0/), which permits unrestricted use, distribution, and reproduction in any medium, provided you give appropriate credit to the original author(s) and the source, provide a link to the Creative Commons license, and indicate if changes were made.

\section{References}

1. Berghoff AS, Preusser M (2018) New developments in brain metastases. Ther Adv Neurol Disord 28(11):1-14

2. Rahmathulla G, Toms SA, Weil RJ (2012) The molecular biology of brain metastasis. J Oncol. https://doi.org/10.1155/2012/723541

3. Nolan C, De Angelis LM (2018) Overview of metastatic disease of the central nervous system. In: Schiff D, Bent MJ (eds) Handbook of clinical neurology, vol 149. Elsevier, Amsterdam, pp 2-23

4. Wen PY, Loeffler JS (2000) Brain metastasis. Curr Treat Options Oncol 1(5):447-458

5. Abbott NJ, Ronnback L, Hansson E (2006) Astrocyte-endothelial interactions at the blood-brain barrier. Nat Rev Neurosci 7(1):41-53

6. Kis B, Chen L, Ueta Y, Busija DW (2006) Autocrine peptide mediators of cerebral endothelial cells and their role in the regulation of blood-brain barrier. J Appl Physiol 27(1):211-222

7. Soto MS, Serres S, Anthony DC, Sibson NR (2014) Functional role of endothelial adhesion molecules in the early stages of brain metastasis. Neuro Oncol 16(4):540-551. https://doi.org/10.1093/ neuonc/not222

8. Fazakas C, Wilhelm I, Nagyoszi P, Farkas AE, Hasko J, Molnar J, Bauer H, Bauer HC, Ayaydin F, Dung NT, Kiklos L, Krizbai IA
(2011) Transmigration of melanoma cells through the blood-brain barrier: role of endothelial tight junctions and melanoma-released serine proteases. PLoS ONE 6(6):e20758

9. Martinez N, Boire A, Deangelis LM (2013) Molecular interactions in the development of brain metastases. Int J Mol Sci 14(8):17157-17167. https://doi.org/10.3390/ijms140817157

10. Miles FL, Pruitt FL, van Golen KL, Cooper CR (2008) Stepping out of the flow: capillary extravasation in cancer metastasis. Clin Exp Metastasis 25(4):305-324

11. Jassam SA, Maherally Z, Smith JR, Ashkan K, Roncaroli F, Fillmore HL, Pilkington GJ (2016) TNF- $\alpha$ enhancement of CD62E mediates adhesion of non-small cell lung cancer cells to brain endothelium via CD15 in lung-brain metastasis. Neuro Oncol 18(5):679-690. https://doi.org/10.1093/neuonc/nov248

12. Jassam SA, Maherally Z, Smith JR, Ashkan K, Roncaroli F, Fillmore HL, Pilkington GJ (2017) CD15s/CD62E interaction mediates the adhesion of non-small cell lung cancer cells on brain endothelial cells: implications for cerebral metastasis. Int J Mol Sci 18(7):1474-1490. https://doi.org/10.3390/ijms18071474

13. Blanas A, Sahasrabudhe NM, Rodríguez E, van Kooyk Y, van Vliet SJ (2018) Fucosylated antigens in cancer: an alliance toward tumor progression, metastasis, and resistance to chemotherapy [published correction appears in Front Oncol. 8:150]. Front Oncol 8:39. https://doi.org/10.3389/fonc.2018.00150

14. Vu K, Weksler B, Romero I, Couraud PO, Gelli A (2009) Immortalized human brain endothelial cell line HCMEC/D3 as a model of the blood-brain barrier facilitates in vitro studies of central nervous system infection by Cryptococcus neoformans. Eukaryot Cell 11:1803-1807

15. An Q, Fillmore HL, Vouri M, Pilkington GJ (2014) Brain tumor cell line authentication, an efficient alternative to capillary electrophoresis by using a microfluidics-based system. Neuro Oncol 16(2):265-273. https://doi.org/10.1093/neuonc/not202

16. Maherally Z, Fillmore HL, Tan SL, Tan SF, Jassam SA, Quack FI, Hatherell KE, Pilkington GJ (2018) Real-time acquisition of transendothelial electrical resistance in an all-human, in vitro, 3-dimensional, blood-brain barrier model exemplifies tight-junction integrity. FASEB J 32(1):168-182. https://doi.org/10.1096/ fj.201700162R

17. Desiderio V, Papagerakis P, Tirino V, Zheng L, Matossian M, Prince ME, Paino F, Mele L, Papaccio F, Montella R, Papaccio G, Papagerakis S (2015) Increased fucosylation has a pivotal role in invasive and metastatic properties of head and neck cancer stem cells. Oncotarget 6(1):71-84

18. Liang J, Liang Y, Gao W (2016) Clinicopathological and prognostic significance of sialyl Lewis $X$ overexpression in patients with cancer: a meta-analysis. OncoTargets Therapy 9:3113-3125. https://doi.org/10.3748/wjg.v20.i20.6123

19. Strell C, Entschladen F (2008) Extravasation of leukocytes in comparison to tumor cells. Cell Commun Signal 4(6):10. https:// doi.org/10.1186/1478-811X-6-10

20. Cailleau-Thomas A, Coullin P, Candelier JJ, Balanzino L, Mennesson B, Oriol R, Mollicone R (2000) FUT4 and FUT9 genes are expressed early in human embryogenesis. Glycobiology 10(8):789-802. https://doi.org/10.1021/mp900178j

21. de Vries T, Knegtel RM, Holmes EH, Macher BA (2011) Fucosyltransferases: structure/function studies. Glycobiology 11(10):119R-128R

22. Mondal N, Dykstra B, Lee J, Ashline DJ, Reinhold VN, Rossi DJ, Sackstein R (2018) Distinct human alpha(1,3)-fucosyltransferases drive Lewis-X/sialyl Lewis-X assembly in human cells. J Biol Chem 293(19):7300-7314. https://doi.org/10.1074/jbc.RA117 .000775

23. Schneider M, Al-Shareffi E, Haltiwanger RS (2017) Biological functions of fucose in mammals. Glycobiology 27(7):601R-618R. https://doi.org/10.1093/glycob/cwx034 
24. Giordano G, Febbraro A, Tomaselli E, Sarnicola ML, Parcesepe P, Parente D, Forte N, Fabozzi A, Remo A, Bonetti A, Manfrin E, Ghasemi S, Ceccarelli M, Cerulo L, Bazzoni F, Pancione M (2015) Cancer-related CD15/FUT4 overexpression decreases benefit to agents targeting EGFR or VEGF acting as a novel RAFMEK-ERK kinase downstream regulator in metastatic colorectal cancer. J Exp Clin Cancer Res 34:108

25. Nakayama F, Nishihara S, Iwasaki H, Kudo T, Okubo R, Kaneko M, Nakamura M, Karube, M, Sasaki K, Narjimatsu H, (2001) CD15 expression in mature granulocytes is determined by alpha 1,3-fucosyltransferase IX, but in promyelocytes and monocytes by alpha 1,3-fucosyltransferase IV. J Biol Chem 276(19):1610016106. https://doi.org/10.1074/jbc.M007272200

26. Homeister JW, Thall AD, Petryniak B, Maly P, Rogers CE, Smith PL, Kelly RJ, Gersten KM, Askari SW, Cheng G, Smithson G, Marks RM, Misra AK, Hindsgaul O, von Andrian UH, Lowe JB (2001) The alpha(1,3)fucosyltransferases FucT-IV and FucT-VII exert collaborative control over selectin-dependent leukocyte recruitment and lymphocyte homing. Immunity 15(1):115-126

27. Barthel SR, Wiese GK, Cho J, Opperman MJ, Hays DL, Siddiqui J, Pienta KJ, Furie B, Dimitroff CJ (2009) Alpha 1,3 fucosyltransferases are master regulators of prostate cancer cell trafficking. Proc Natl Acad Sci USA 106(46):19491-19496. https://doi. org/10.1073/pnas.0906074106

28. Jorgensen T, Berner A, Kaalhus O, Tveter KJ, Danielsen HE, Bryne M (1995) Up-regulation of the oligosaccharide sialyl LewisX: a new prognostic parameter in metastatic prostate cancer. Cancer Res 55(9):1817-1819
29. Li J, Guillebon AD, Hsu JW, Barthel SR, Dimitroff CJ, Lee YF, King MR (2013) Human fucosyltransferase 6 enables prostate cancer metastasis to bone. Br J Cancer 109:3014-3022. https:// doi.org/10.1038/bjc. 2013.690

30. Ogawa J, Inoue H, Koide S (1996) Expression of alpha-1,3-fucosyltransferase type IV and VII genes is related to poor prognosis in lung cancer. Cancer Res 56(2):325-329

31. Yang X, Liu S, Yan Q (2013) Role of fucosyltransferase IV in epithelial-mesenchymal transition in breast cancer cells. Cell Death Dis 25(4):e735. https://doi.org/10.1038/cddis.2013.241

32. Martin K, Akinwunmi J, Rooprai HK, Kennedy AJ, Linke A, Ognjenovic N, Pilkington GJ (1995) Nonexpression of CD15 by neoplastic glia: a barrier to metastasis? Anticancer Res 15(4):1159-1166

33. Zhao ZR, Lau RWH, Long H, Mok TSK, Chen GG, Underwood MJ, Ng CSH (2018) Novel method for rapid identification of micropapillary or solid components in early-stage lung adenocarcinoma. J Thorac Cardiovasc Surg 156(6):2310-2318. https:// doi.org/10.1016/j.jtcvs.2018.07.054

Publisher's Note Springer Nature remains neutral with regard to jurisdictional claims in published maps and institutional affiliations 